\title{
Avaliação da Gerência de Incidentes sob a Luz do MR-MPS-SV e Medição para Apoiar a Melhoria da Qualidade do Serviço de TI
}

\author{
Bianca Trinkenreich, Gleison Santos
}

Programa de Pós-Graduação em Informática, Departamento de Informática Aplicada, Universidade Federal do Estado do Rio de Janeiro (UNIRIO), Av. Pasteur, RJ, Brasil

\{bianca.trinkenreich, gleison.santos\}@uniriotec.br

Resumo. O objetivo principal do gerenciamento de incidentes é restaurar o nível de serviço o mais rápido possível. Prestadores de serviços buscam continuamente métodos eficientes para medir e gerenciar seus serviços. Um processo de medição adequado ajuda a compreender as capacidades e desenvolver planos viáveis para entregar os serviços dentro da qualidade acordada. Este artigo apresenta a avaliação do processo de Gerência de Incidentes de uma empresa global de grande porte, sob a luz do MR-MPS-SV, e propõe um processo para seleção de medidas multiprocessos a partir do mapeamento e dos objetivos da organização para apoiar a melhoria de serviços de TI.

Resumo. Main goal of incident management is restore the service level as soon as possible. Service providers are continually aiming for more efficient methods to measure and manage services. A good measurement process helps understanding capabilities and developing feasible plans to deliver services according to expected quality. This paper presents an Incident Management process evaluation in a global company, under the light of MR-MPS-SV, and suggests a process to select measures related to more than one process, from organization goals and process mapping to support IT services improvement.

\section{Introdução}

Serviço é uma forma de entregar valor aos clientes, facilitando o alcance dos resultados desejados sem custos e riscos associados aos detalhes específicos da execução. A prática do gerenciamento de serviços de TI tem crescido através de uma abordagem orientada a serviços de gerenciamento integrado de TI, dando suporte às camadas de software, infraestrutura e processos [TSO, 2011].

Referências e diretrizes sobre como desenvolver e melhorar a maturidade do serviço prestado são alicerces para melhorar o desempenho do serviço e satisfação do cliente [Forrester et al., 2010]. Os modelos de maturidade de serviços MR-MPS-SV [Softex, 2012a] e CMMI-SVC [Forrester et al., 2010] foram criados para atender esta necessidade. Os preceitos de tais modelos podem complementar modelos de maturidade de software para estruturar o desenvolvimento de software como um serviço de TI [Kalinowski e Reinerh, 2013] e obter benefícios em relação à qualidade, prazo, custo e satisfação do cliente em relação à entrega do software. Os modelos de maturidade organizam a prestação de serviços em diversos processos, que precisam ser executados e medidos de forma integrada para que o serviço seja entregue pelos fornecedores a seus clientes dentro dos níveis acordados de qualidade. 
O Modelo de Referência MPS para Serviços (MR-MPS-SV) [Softex, 2012a] tem o foco principalmente nas micro, pequenas e médias empresas que prestam serviços de TI. A definição dos processos se baseia na ISO/IEC 15504-2, ISO/IEC 20000, MRMPS-SW [Softex, 2012b] e CMMI-SVC [CMMI Product Team, 2010]. O MR-MPS-SV é formado por 7 níveis de maturidade (do $\mathrm{G}$ ao A) e 24 processos, 12 deles baseados na norma ISO/IEC 20000, próprios de serviços e sem equivalência no MR-MPS-SW.

O objetivo do processo Gerência de Incidentes (GIN), nível G do MR-MPS-SV, é restaurar os serviços aos níveis acordados e cumprir as solicitações de acordo com os Acordos de Nível de Serviço (ANS) entre o fornecedor e seus clientes [Softex, 2012c]. Incidente é "uma interrupção não planejada de um serviço, uma redução na qualidade de um serviço ou um evento que ainda não impactou o serviço para o cliente" [ISO/IEC, 2011]. Gerenciar um incidente inclui registrar, acompanhar, escalonar (se necessário), e encerrar o incidente. O gerenciamento de incidentes é altamente visível para o negócio, e mais fácil de demonstrar seu valor do que a maioria dos demais processos de serviços. Por esta razão, ele é muitas vezes um dos primeiros processos a serem implantados em projetos de gestão de serviços [TSO, 2011], e muitas vezes concentra a maior quantidade de medidas utilizadas para controle e gestão da qualidade do serviço [Trinkenreich et al., 2015]. Este processo pode ser usado para destacar outras áreas que precisam de atenção, proporcionando, assim, uma justificativa para as despesas de implantação dos demais processos de serviços [TSO, 2011].

Considerando o provimento de serviços como uma cadeia de processos relacionados, mais do que selecionar os processos a serem medidos e entregar apenas medidas isoladas de cada processo, também é preciso conhecer e controlar estes relacionamentos. Para isso, é necessário saber derivar adequadamente as medidas operacionais para gerenciar o relacionamento existente entre os processos, e avaliar o impacto de um processo em outro (que estamos chamando de "medidas multiprocessos").

Este artigo apresenta um relato de experiência sobre a avaliação do processo de Gerência de Incidentes de uma empresa global de grande porte, com objetivo de apoiar a seleção de medidas usadas nos indicadores de qualidade. A avaliação do processo atual foi guiada à luz dos resultados esperados do processo Gerência de Incidentes (GIN) do modelo de maturidade de serviços MS-MPS-SV, com foco nas interseções deste processo com outros. O processo de seleção de medidas proposto foi baseado no GQM+Strategies [Basili et al, 2007]. Após a introdução, o artigo apresenta a descrição do estudo de caso realizado (seção 2) e as considerações finais (seção 3).

\section{Descrição da Experiência de Aplicação do Estudo de Caso}

Um método de estudo de caso é uma técnica de pesquisa exploratória usada para destacar e explorar aspectos que podem guiar respostas à questão de pesquisa. O método é relevante para Sistemas de Informação quando o pesquisador consegue estudar o sistema de informação em um ambiente real, e assim é capaz de responder perguntas do tipo "como" e "por quê", e quando não há uma grande quantidade de pesquisas formais conduzidas previamente sobre o assunto abordado [Recker, 2013].

A experiência relatada aconteceu na área de Tecnologia de Informação de uma empresa global de grande porte (chamada de Organização A), que executa seus processos seguindo as práticas da biblioteca ITIL [TSO, 2011b]. A Organização A não é avaliada em nenhum modelo de maturidade de software ou serviços. O propósito da experi- 
ência foi utilizar os resultados esperados do processo Gerência de Incidentes (GIN) do MR-MPS-SV para guiar a avaliação da qualidade do processo atual, avaliar os relacionamentos deste com outros processos, avaliar as medidas utilizadas e apoiar a organização com a proposta de ações de melhoria do processo e das medidas. A motivação desta experiência ocorreu como continuação do trabalho [Trinkenreich e Santos, 2014] que availou o processo de medição (MED) e a qualidade de duas medidas utilizadas pela organização, uma delas relacionada ao processo de Gerência de Incidentes (GIN) que não conseguia atender ao objetivo da organização. A avaliação mais detalhada deste processo foi sugerida como parte dos trabalhos futuros.

\subsection{Avaliação do Processo de Gerência de Incidentes}

A avaliação de aderência do processo GIN ao modelo de maturidade MR-MPS-SV está resumida na Tabela 1 e mostra como a Organização A atende cada resultado esperado.

Tabela 1 - Resultados esperados do processo GIN na Organização A

\begin{tabular}{|l|}
\hline \multicolumn{1}{|c|}{ Resultado esperado } \\
\hline GIN 1 - Uma abordagem para \\
o gerenciamento de incidentes \\
e solicitação de serviço é esta- \\
belecida e mantida.
\end{tabular}

belecida e mantida.

GIN 2 - Um sistema de gerenciamento e controle de incidentes e solicitação de serviços é estabelecido e mantido.

GIN 3 - Incidentes e solicitações de serviços são registrados e classificados

GIN 4 - Incidentes e solicitações de serviços são priorizados e analisados

GIN 5 - Incidentes e solicitações de serviços são resolvidos e encerrados

GIN 6 - Incidentes e solicitações de serviços que não progrediram conforme acordos de nível de serviço são escalonados.

GIN 7 - Informações a respeito da situação ou progresso de um incidente relatado ou solicitação de serviço são comunicadas às partes interessadas

\section{Como a Organização A atende o resultado esperado}

Documentação estruturada e detalhada, descrevendo o processo de gerência de incidentes, que é composto por sete subprocessos principais para tratamento de requisições de usuários, segundo a Figura 1 e a Tabela 2.

O sistema de gerenciamento de incidentes usado é baseado na solução

BMC Remedy (http://www.bmc.com/it-solutions/remedy-itsm.html), integrado com e-mail e mensagens instantâneas para facilitar as comunicações e o atendimento. O sistema também é usado pelos demais processos que se integram com a Gerência de Incidentes, gerando uma visão integrada do fluxo.

Em "1. Registro do Incidente", o service-desk recebe requisição de incidente, verifica se há incidente anterior, coleta as informações e registra o novo incidente. Se o service-desk for capaz de resolver a solicitação usando a base de conhecimento, o incidente é encerrado. Senão, é classificado em relação ao Impacto (extensão do impacto do incidente para os usuários e criticidade para o negócio) e Urgência (quão urgente a resolução do incidente é para o usuário e os efeitos sobre os processos de negócios).

De acordo com as associações pré-definidas entre Impacto (1 - Extenso, 2 - Grande, 3 - Moderado ou 4 - Pequeno) e Urgência (1 - Crítico, 2 - Alto, 3 - Médio ou 4 - Baixo), a classificação de prioridade do incidente é feita. Quando o service-desk recebe a solução do especialista e é capaz de resolver um incidente, o subprocesso "6. Encerramento do Incidente" é seguido. O service-desk atualiza e fecha o incidente com a solução enviada pelo especialista e disponibiliza o incidente para o usuário validar a solução, executando o subprocesso "7. Validação da Solução".

Quando um incidente tem Impacto "1-Extenso" e Urgência "1 - Crítico", ele é escalonado para situações de crise, seguindo o subprocesso "5. Tratamento de Escalonamento de Incidente". Gerência de crises faz parte do processo GCD, com uma conferência realizada com todas as equipes técnicas necessárias para restabelecer o serviço.

Ao receber a notificação de conclusão do incidente, o usuário verifica a solução. Se for aceitável, não é preciso tomar nenhuma ação (em 5 dias o incidente é encerrado automaticamente). Se o usuário não considerar a solução aceitável, ele pode entrar no sistema e reabrir o incidente, solicitando uma melhor solução.

Tabela 2 - Subprocessos de Gerência de Incidentes

\begin{tabular}{|l|l|}
\hline Subprocessos & \multicolumn{1}{c|}{ Objetivo } \\
\hline Registro de & Utilizado por analistas da equipe de service-desk quando recebem as requisições e \\
\hline
\end{tabular}




\begin{tabular}{|c|c|}
\hline Subprocessos & Objetivo \\
\hline Incidente & registram pedidos de incidentes feitos pelos usuários. \\
\hline $\begin{array}{l}\text { Atribuição de } \\
\text { Incidente }\end{array}$ & $\begin{array}{l}\text { Utilizado por analistas da equipe de service-desk e por coordenadores para atribuir } \\
\text { pedidos de incidentes aos especialistas adequados ou alterar coordenadores para a } \\
\text { resolução ou implementação. }\end{array}$ \\
\hline $\begin{array}{l}\text { Rastreamento de } \\
\text { Incidente }\end{array}$ & $\begin{array}{l}\text { Utilizado por coordenadores quando estão lidando com notificações de redesignação } \\
\text { ou escalonamento de SLA. }\end{array}$ \\
\hline $\begin{array}{l}\text { Resolução de } \\
\text { Incidente }\end{array}$ & $\begin{array}{l}\text { Utilizado por especialistas na resolução de solicitações de incidentes que foram atri- } \\
\text { buídos a eles. }\end{array}$ \\
\hline $\begin{array}{l}\text { Tratamento de } \\
\text { Escalonamento }\end{array}$ & $\begin{array}{l}\text { Pode ser utilizado pelo proprietário do serviço afetado para que o incidente seja ser } \\
\text { resolvido da forma mais eficiente. }\end{array}$ \\
\hline $\begin{array}{l}\text { Encerramento de } \\
\text { Incidente }\end{array}$ & Utilizado por analistas da equipe de service-desk quando podem resolver incidentes. \\
\hline $\begin{array}{l}\text { Validação da } \\
\text { Solução }\end{array}$ & $\begin{array}{l}\text { Utilizado pelo usuário solicitante após revisar e aceitar os pedidos de encerramento de } \\
\text { seus incidentes. }\end{array}$ \\
\hline
\end{tabular}

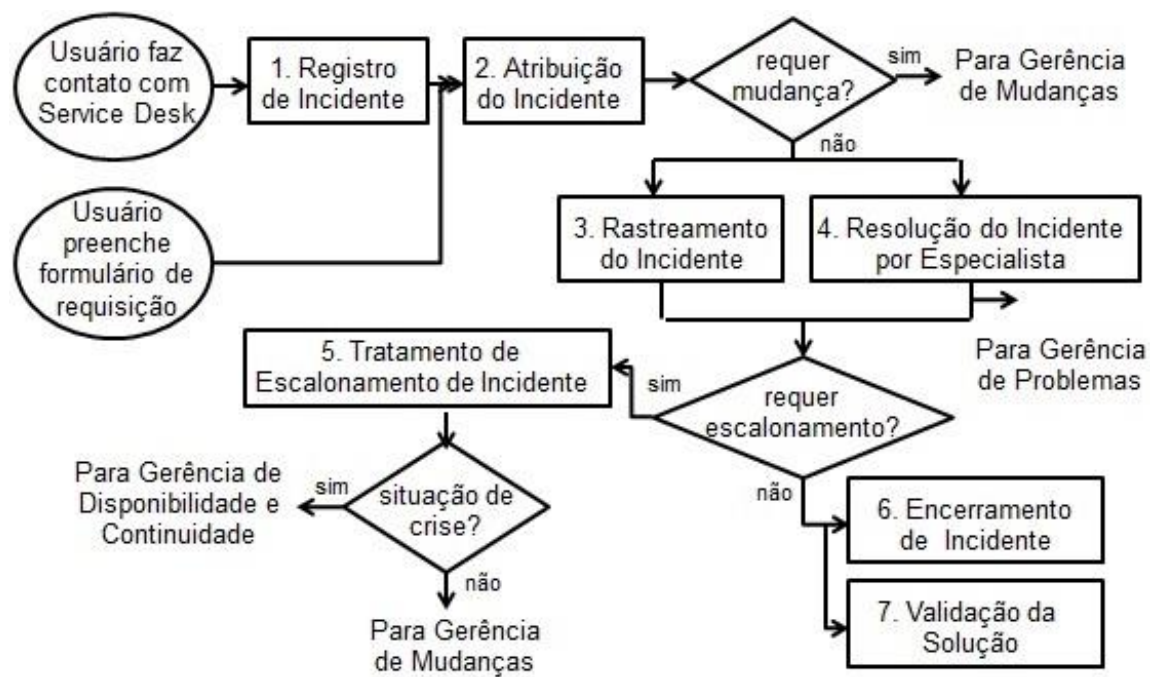

Figura 1 - Macro-processo de Gerência de Incidentes na organização estudada

\subsection{Criação de um processo para seleção de medidas}

As três medidas sobre o processo de Gerência de Incidentes (GIN) utilizadas pela organização são: "Percentual de incidentes resolvidos dentro do prazo acordado no ANS", "Percentual de Incidentes Resolvidos pelo Primeiro Nível" e "Percentual de Incidentes Não-Resolvidos". O gerente da área de Incidentes informou que o objetivo da área é reduzir o tempo de resolução de incidentes. O processo de Gerência de Incidentes (GIN) é aquele para o qual mais se encontram medidas de serviços na literatura, e as três medidas utilizadas pela organização haviam sido identificadas em um mapeamento sistemático conduzido pelos autores [Trinkenreich et al., 2015].

Medidas operacionais são usadas pelas organizações de TI, mas não respondem perguntas sobre a eficiência geral no provimento de serviços de TI, como outras medidas poderiam ajudar a fazê-lo, por exemplo: "Taxa de incidentes abertos devido a mudanças" [Steinberg, 2001]. Esta medida relaciona os processos Gerência de Incidentes (GIN) e Gerência de Mudanças (GMU), e poderia ser utilizada pela organização em questão para avaliar as causas de abertura de incidentes. Desta forma, seria possível apoiar o entendimento de uma possível relação causa-efeito entre os processos, como os cenários de mudanças executadas (como implantação de novo hardware, atualização de 
sistema etc.) e o aumento ou diminuição de incidentes abertos (GIN).

O gerente da equipe de infraestrutura, parte da diretoria de Serviços de TI, informou que as maiores dificuldades enfrentadas no processo de medição de serviços são: (i) desdobramento dos objetivos estratégicos em objetivos de serviços de TI, (ii) seleção de estratégias para atender os objetivos de serviços de TI, (iii) mudança e falta de controle dos fatores de contexto e premissas após a seleção de estratégias e medidas. Para facilitar a seleção de medidas, atendendo às dificuldades informadas, foi criado um novo processo conforme a Figura 2. Este processo foi baseado na abordagem GQM+Strategies [Basili et al., 2007], reuso de medidas [Gencel et al., 2013] e o mapeamento dos relacionamentos entre processos de serviços de TI da seção anterior.

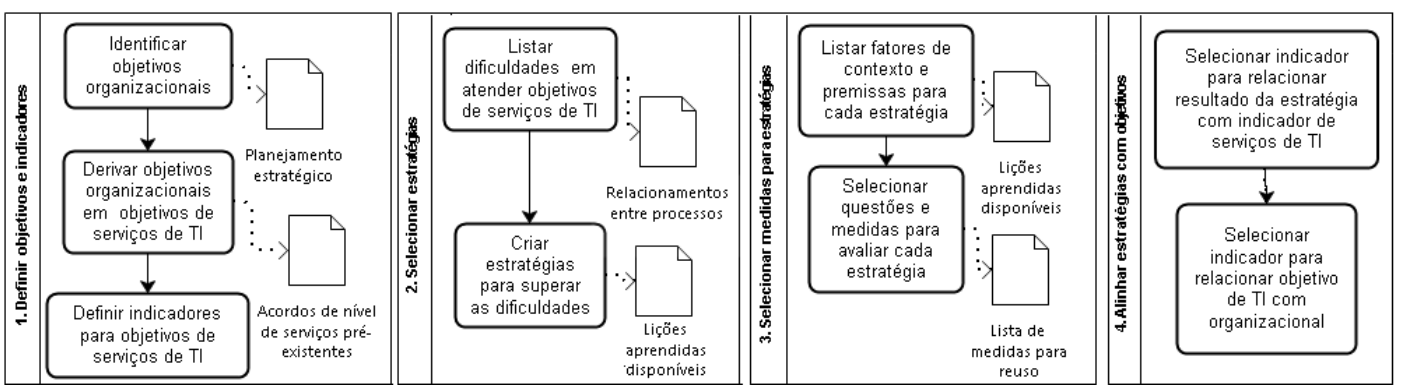

Figura 2 - Processo proposto para seleção de medidas de serviços de $\mathrm{TI}$

A abordagem GQM+Strategies [Basili et al., 2007] é uma extensão do GQM [Solingen e Berghout, 1999], e utiliza estratégias e medidas como dados de entrada para um modelo do nível mais alto de negócio até o mais baixo de operação, e vice-versa. As estratégias para cada objetivo são usadas como planos diferentes para tipos diferentes de objetivos. Os componentes desta abordagem são: Objetivo organizacional (o que se deseja alcançar), Estratégia (como alcançar o objetivo), Fator de Contexto (variável de ambiente interna ou externa que afeta a estratégia e as medidas), Premissas (suposições desconhecidas que podem afetar a estratégia e as medidas), Gráfico GQM (como medir se o objetivo foi alcançado e se a estratégia foi bem sucedida) e Modelo de Interpretação (apoio para interpretar os dados).

Anualmente a definição dos objetivos organizacionais da Organização A acontece sem a participação dos gerentes de TI, que já recebem os objetivos da diretoria e definem a partir deles os objetivos de TI. O objetivo organizacional repassado para a TI em 2015 foi de "Melhorar Experiência do Usuário com a TI", que é medido através dos acordos de nível de serviço, que incluem tempo de resolução de incidentes e requisições, percentual de disponibilidade dos serviços, entre outros, e é medido por meio do indicador "Percentual de Atingimento dos itens do Acordo de Nível de Serviço".

Os acordos de nível de serviços foram avaliados e verificou-se que um dos itens não atendidos em 2014 foi o tempo de resolução de incidentes. Com isso, o primeiro objetivo definido pela área de serviços de TI foi "Reduzir o Tempo de Solução de Incidentes", cujo desdobramento seguindo o processo na Figura 2 é ilustrado na Figura 3.

O mapeamento dos relacionamentos entre o processo de Incidentes e os processos de Mudanças, Problemas e Disponibilidade foi usado na análise de causas e dificuldades para reduzir o tempo de resolução dos incidentes. Os problemas encontrados nas relações de causa-efeito entre os processos não são as únicas causas para os incidentes terem sido solucionados fora do tempo acordado. O diagrama da Figura 3 mostra apenas 
estratégias selecionadas a partir destas relações, conforme o escopo desta experiência.

Foram criadas estratégias (Tabela 3) com medidas operacionais para as dificuldades encontradas nos relacionamentos descritos acima e indicadores para medir a contribuição de cada uma destas estratégias para o objetivo de serviços de TI.



Figura 3 - Seleção de medidas: "Reduzir tempo de resolução de incidentes" 
A consulta de medidas em uma biblioteca foi parte do método apresentado por Kilpi (2001) na Nokia. Na experiência relatada no presente artigo, consultamos uma base de medidas classificada por processo do MR-MPS-SV e do CMMI-SVC [Trinkenreich et al, 2015], para reuso de medidas relacionadas ao processo Gerência de Incidentes abordado. Dentre as medidas deste processo, estão: "Tempo médio entre incidentes", "Tempo médio para restaurar um sistema", "Quantidade de incidentes reincidentes, escalonados e redirecionados", "Tempo médio para registrar, categorizar, priorizar, e resolver um incidente", "Quantidade de incidentes atendidos no prazo", "Tempo médio de resposta por equipe", "Percentual de incidentes com descrição correta", "Percentual de incidentes fechados pelo service desk", dentre outras.

Tabela 3 - Estratégias, medidas e indicadores multiprocessos

\begin{tabular}{|c|c|c|c|c|}
\hline Estratégias & Descrição & Medidas & Indicadores & Processos \\
\hline $\begin{array}{l}\text { 1. Melhorar o } \\
\text { processo de } \\
\text { aprovação de } \\
\text { mudanças } \\
\text { corretivas }\end{array}$ & $\begin{array}{l}\text { Uma mudança corretiva é realizada caso } \\
\text { seja necessário fazer uma alteração para } \\
\text { solução do incidente. O processo de mu- } \\
\text { danças inclui planejamento, criação e apro- } \\
\text { vação por um comitê e o tempo de execu- } \\
\text { ção destas atividades deve ser alinhado ao } \\
\text { tempo acordado para solução do incidente. }\end{array}$ & $\begin{array}{l}\text { Tempo médio de } \\
\text { aprovação de } \\
\text { mudanças } \\
\text { corretivas }\end{array}$ & $\begin{array}{l}\text { Percentual de } \\
\text { incidentes } \\
\text { atendidos fora } \\
\text { do prazo que } \\
\text { demandaram } \\
\text { mudanças } \\
\text { corretivas }\end{array}$ & $\begin{array}{l}\text { Gerência de } \\
\text { Incidentes e } \\
\text { Gerência de } \\
\text { Mudanças }\end{array}$ \\
\hline $\begin{array}{l}\text { 2. Melhorar o } \\
\text { processo de } \\
\text { resolução de } \\
\text { problemas } \\
\text { para evitar } \\
\text { incidentes } \\
\text { repetidos } \\
\end{array}$ & $\begin{array}{l}\text { Se a solução aplicada for de contorno e o } \\
\text { incidente puder se repetir, um registro de } \\
\text { problema deve ser criado para investigação } \\
\text { de causa-raiz. Erros recorrentes sobrecarre- } \\
\text { gam as equipes de atendimento e contribu- } \\
\text { em para aumento do tempo de solução dos } \\
\text { incidentes no total. }\end{array}$ & $\begin{array}{l}\text { Quantidade de } \\
\text { problemas abertos } \\
\text { para erros } \\
\text { conhecidos e Per- } \\
\text { centual de pro- } \\
\text { blemas } \\
\text { solucionados }\end{array}$ & $\begin{array}{l}\text { Percentual de } \\
\text { incidentes } \\
\text { recorrentes }\end{array}$ & $\begin{array}{l}\text { Gerência de } \\
\text { Incidentes e } \\
\text { Gerência de } \\
\text { Problemas }\end{array}$ \\
\hline $\begin{array}{l}\text { 3. Melhorar o } \\
\text { processo de } \\
\text { escalonamen- } \\
\text { to de inciden- } \\
\text { tes graves }\end{array}$ & $\begin{array}{l}\text { Se o incidente for classificado com "Impac- } \\
\text { to" } 1 \text {-Extenso e "Urgência" } 1 \text { - Critico, ele } \\
\text { é escalonado para crise. Uma reunião com } \\
\text { todos os representantes das equipes que dão } \\
\text { suporte ao serviço (previamente documen- } \\
\text { tado) é realizada e fica aberta até que o } \\
\text { nível de serviço seja restabelecido. }\end{array}$ & $\begin{array}{l}\text { Tempo médio de } \\
\text { escalonamento de } \\
\text { incidente à crise e } \\
\text { Tempo médio de } \\
\text { solução de crises }\end{array}$ & $\begin{array}{l}\text { Percentual de } \\
\text { incidentes } \\
\text { atendidos fora } \\
\text { do prazo que } \\
\text { foram } \\
\text { escalonados } \\
\text { para crise }\end{array}$ & $\begin{array}{l}\text { Gerência de } \\
\text { Incidentes e } \\
\text { Gerência de } \\
\text { Continuida- } \\
\text { de e Dispo- } \\
\text { nibilidade }\end{array}$ \\
\hline
\end{tabular}

A medida "Quantidade de incidentes reincidentes, escalonados e redirecionados" foi desdobrada para o relacionamento de Gerência de Incidentes com ambas Gerência de Problemas e Gerência de Continuidade e Disponibilidade, uma vez que incidentes reincidentes são aqueles que devem ter investigação de causa-raiz e incidentes escalonados são aqueles para os quais é aberta uma situação de crise.

\section{Conclusões}

A experiência relatada neste artigo foi realizada em uma empresa global de grande porte avaliando a Gerência de Incidentes sob a luz dos resultados esperados do MS-MPS-SV e as medidas em uso pela organização. Os objetivos de pesquisa foram alcançados. Através do mapeamento da Gerência de Incidentes, foi possível encontrar seu relacionamento com os demais processos de serviços, e estratégias relacionadas a dificuldades nestas integrações. Como contribuição, um processo para seleção de medidas e indicadores de qualidade multiprocessos para controlar os relacionamentos existentes entre os processos e em alinhamento o objetivo estratégico do negócio foi criado e aplicado.

O processo foi criado para selecionar medidas para Gerência de Incidentes, que permeia todas as áreas de serviços de TI da organização (Infraestrutura, Aplicações, 
Telefonia e Usuários Finais). Ele foi avaliado pelo gerente de Infraestrutura, que informou que o processo atende às necessidades da área e solicitou que seja aplicado para desdobramento dos demais objetivos e que inclua um sistema automatizado para entrada dos dados coletados e cálculo dos indicadores (a ser tratado em trabalho futuro).

O uso do GQM+Strategies ajudou a organização a desdobrar um objetivo organizacional em objetivo de serviços de TI, antes de criar as estratégias, e possibilitou diminuir o nível de abstração dos objetivos com ações mais concretas e operacionais. A validação do alinhamento de indicadores no último passo do processo foi proposta para que a granularidade dos níveis não causasse distanciamento do objetivo organizacional. Constatou-se que o reuso de medidas, através de consulta em uma biblioteca, facilitou a seleção de medidas e indicadores. Também verificou-se que os acordos de nível de serviço são artefatos capazes de traduzir uma medida qualitativa como "satisfação do usuário" em indicadores quantitativos acordados entre as partes que podem ser mensuráveis e gerenciados, tornando mais direta a seleção dos indicadores de serviços de TI.

\section{Agradecimentos}

Os autores agradecem à FAPERJ (projeto E-26/110.438/2014) pelo auxílio financeiro.

\section{Referências}

Basili, V., Heidrich, J., Lindvall, M., Münch, J., Regardie, M., Trendowicz, A. (2007) "GQM+Strategies - aligning business strategies with software measurement". International Symposium on Empirical Software Engineering and Measurement, Spain, pp 488-490.

Forrester, E., Buteau, B., Shrum, S. (2010) -CMMI For Services, Guidelines for Superior Service. CMMI-SVC Version 1.3 - 2nd Edition. SEI. Addison-Wesley Professional.

Kilpi, T. (2001) - "Implementing a software metrics program at Nokia", IEEE Software, (Volume18, issue 6, pp. 72-77. ISSN: 0740-7459

Kalinowski, M., Reinehr, S. (2013) "Estruturando Desenvolvimento de Software como um Serviço de TI: Uma Experiência Prática" - Simpósio Brasileiro de Qualidade de Software, Salvador - BA, pp. 318-325

Recker, J., 2013. "Scientific Research in Information Systems - A Beginner's Guide". Springer, ISBN 978-3-642-30048-6

Softex (2012a) Associação para Promoção da Excelência do Software Brasileiro "MPS.BR Guia Geral MPS de Serviços". Disponível em www.softex.br

Softex (2012b) - Associação para Promoção da Excelência do Software Brasileiro "MPS.BR Guia Geral MPS de Software". Disponível em www.softex.br.

Softex (2012c) Associação para Promoção da Excelência do Software Brasileiro - "Guia de Implementação - Parte 1: Fundamentação para Implementação do Nível G do MR-MPSSV:2012". Disponível em www.softex.br.

Solingen, R., Berghout, E. (1999) "The Goal/Question/Metric Method: A Practical Guide for Quality Improvement of Software Development”. McGraw-Hill.

TSO - The Stationery Office (2011). "ITIL Service Operations" - Information Technology Infrastructure Library, London.

Steinberg, R. (2001) "Measuring ITSM: Measuring, Reporting, and Modeling the IT Service Management Metrics that Matter Most to IT Senior Executives". Ed. Trafford.

Trinkenreich, B., Santos, G. (2014) "Avaliação do Processo de Medição em Gerência de Incidentes e Gerência de Continuidade e Disponibilidade à Luz do MR-MPS-SV". Workshop Anual do MPS.BR - WAMPS, Campinas - SP, pp. 186-194 .

Trinkenreich, B., Santos, G., Barcellos, M. P. (2015) "Metrics to Support IT Service Maturity Models - A Systematic Mapping Study", 17th International Conference on Enterprise Information Systems (ICEIS), Barcelona, Spain. 